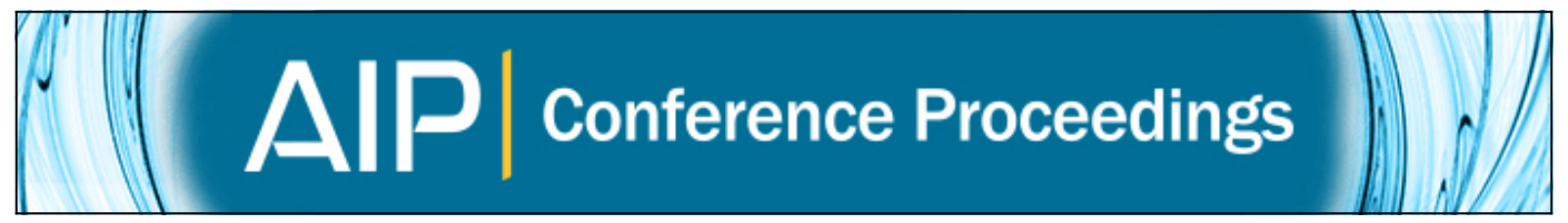

A comparative study of three new conjugate gradient methods with exact line search

Mohamed Hamoda, Mohd Rivaie, Abdelrhaman Abshar, and Mustafa Mamat

Citation: AIP Conference Proceedings 1682, 020030 (2015); doi: 10.1063/1.4932439

View online: http://dx.doi.org/10.1063/1.4932439

View Table of Contents: http://scitation.aip.org/content/aip/proceeding/aipcp/1682?ver=pdfcov

Published by the AIP Publishing

Articles you may be interested in

Global convergence analysis of a new nonlinear conjugate gradient coefficient with strong Wolfe line search

AIP Conf. Proc. 1660, 050014 (2015); 10.1063/1.4915647

A new convergent conjugate gradient method under the exact line search

AIP Conf. Proc. 1660, 050013 (2015); 10.1063/1.4915646

A comparative study of two new conjugate gradient methods

AIP Conf. Proc. 1643, 616 (2015); 10.1063/1.4907502

A new conjugate gradient method with sufficient descent without any line search for unconstrained optimization AIP Conf. Proc. 1643, 602 (2015); 10.1063/1.4907500

A new conjugate gradient method and its global convergence under the exact line search

AIP Conf. Proc. 1635, 639 (2014); 10.1063/1.4903649 


\title{
A Comparative Study of Three New Conjugate Gradient Methods with Exact Line Search
}

\author{
Mohamed Hamoda $^{\mathrm{a}}$, Mohd Rivaie $^{\mathrm{b}}$, Abdelrhaman Abshar $^{\mathrm{c}}$, Mustafa Mamat ${ }^{\mathrm{d}}$ \\ a,c School of Informatics and Applied Mathematics, Universiti Malaysia Terengganu, 21030 Terengganu, Malaysia \\ ahmuda@yahoo.com, ${ }^{\mathrm{c}}$ tomcatkassala@yahoo.com \\ ${ }^{\mathrm{b}}$ Department of Computer Science and Mathematics, Univesiti Teknologi MARA ,23000 Terengganu, Malaysia \\ rivaie75@yahoo.com \\ ${ }^{\mathrm{d}}$ Department of Computational \& Applied Mathematics, Faculty of Informatics and Computing, Universiti Sultan \\ Zainal Abidin, 22200 Terengganu, Malaysia \\ dmust@uniza.edu.my
}

\begin{abstract}
Conjugate Gradient methods play an important role in solving unconstrained optimization, especially for large scale problems. In this paper, we compared the performance profile of the classical conjugate gradient coefficients $F R, P R P$ with three new $\beta_{k}$. These three new $\beta_{k}$ possess global convergence properties using the exact line search. Preliminary numerical results show that the three new $\beta_{k}$ are very promising and efficient when compared to CG coefficients $F R, P R P$.
\end{abstract}

Keywords: Conjugate gradient method, exact line search, global convergence, large scale, unconstrained optimization. PACS: 02.60.Pn, 02.60.Cb, 02.70.C

\section{INTRODUCTION}

Consider the unconstrained optimization problem:

$$
\min f(x) \quad, x \in R^{n}
$$

where $f: R^{n} \rightarrow R$ be bounded from below and a continuously differentiable function, and $R^{n}$ denotes an $\mathrm{n}$ dimensional Euclidean space. We use $g(x)$ to denote the gradient of $f$ at $x$. Conjugate gradient methods are efficient for solving (1), especially when the dimension $n$ is large. The conjugate gradient method for solving (1) generates a sequence of iterates by letting,

$$
x_{k+1}=x_{k}+\alpha_{k} d_{k}, \quad k=0,1,2, \ldots
$$

where $x_{k}$ is current iterate point and $\alpha_{k}$ is a step length, which is computed by carrying out some line search, and $d_{k}$ is the search direction defined by

$$
d_{k}=\left\{\begin{array}{lc}
-g_{k} & \text { if } \quad k=0, \\
-g_{k}+\beta_{k} d_{k-1} & \text { if } \quad k \geq 1,
\end{array}\right.
$$

where $g_{k}=g\left(x_{k}\right)$ and $\beta_{k}$ is a scalar. If the line search is exact, the step length $\alpha_{k}$ is obtained in the direction $d_{k}$ by the rule

$$
\alpha_{k}=\underset{\alpha \geq 0}{\arg \min } f\left(x_{k}+\alpha d_{k}\right)
$$

Well-known conjugate gradient methods include the Hestenes-Stiefel (HS) [1], Fletcher - Reeves $(F R)$ [2], Polak-Ribiere-Polyak $(P R P)$ [3,4], conjugate descent $(C D)$ [5], Liu - Storey $(L S)$ [6], and Dai Yuan $(D Y)$ [7]. The parameters $\beta_{k}$ of these methods are specified as follows:

$$
\begin{aligned}
& \beta_{k}^{H S}=\frac{g_{k}^{T}\left(g_{k}-g_{k-1}\right)}{\left(g_{k}-g_{k-1}\right)^{T} d_{k-1}}, \beta_{k}^{F R}=\frac{g_{k}^{T} g_{k}}{g_{k-1}^{T} g_{k-1}}, \beta_{k}^{P R P}=\frac{g_{k}^{T}\left(g_{k}-g_{k-1}\right)}{g_{k-1}^{T} g_{k-1}}, \beta_{k}^{C D}=-\frac{g_{k}^{T} g_{k}}{d_{k-1}^{T} g_{k-1}}, \quad \beta_{k}^{L S}=-\frac{g_{k}^{T}\left(g_{k}-g_{k-1}\right)}{d_{k-1}^{T} g_{k-1}}, \\
& \beta_{k}^{D Y}=\frac{g_{k}^{T} g_{k}}{\left(g_{k}-g_{k-1}\right)^{T} d_{k-1}}
\end{aligned}
$$


You can download the full-text paper from this website:

http://scitation.aip.org/content/aip/proceeding/aipcp/10.1063/1.4932439?emailalert=true 


\section{REFERENCES}

1. M. R. Hestenes, E. Stiefel. Method of conjugate gradient for solving linear equations, J. Res. Nat. Bur.Stand. 49, (1952), pp. 409-436

2. R. Fletcher, C. Reeves. Function minimization by conjugate gradients, Compute. J. 7, (1964), pp. 149-154.

3. B.T. Polyak. The conjugate gradient method in extreme problems, USSR Comp. Math. Phys. 9, (1969), pp. 94-112.

3. E. Polak, G. Ribie`re. Note Sur la convergence de directions conjuge'es, Rev. Francaise Informat Recherche Operationelle, 3e Anne`e $16,(1969)$, pp. 35-43.

5. Fletcher. Practical Method of Optimization, second ed. Unconstrained Optimization, vol. I, Wiley, New York,(1997).

6. Y. Liu, C. Storey. Efficient generalized conjugate gradient algorithms, part 1: theory, J. Optim.Theory Appl. 69, (1992), pp. 129-137.

7. Y. Dai, Y. Yuan. A nonlinear conjugate gradient with strong global convergence properties, SIAM J. Optim. 10, (2000), pp. $177-182$.

8. G. Zoutendijk, Nonlinear Programming, in: J. Abadie (Ed.), Computational Methods, Integer and Nonlinear Programming, North-Holland, Amsterdam, (1970), pp. 37-86.

9. M.J.D. Powell, Non-convex minimization calculation and the conjugate gradient method, Lecture Notes in Mathematics, 1066,Springer-Verlag, Berlin, (1984), pp. 122- 241.

10. M.J.D. Powell. Restart procedure for the conjugate gradient method, Math. Prog. 2, (1977), pp. 241-254.

11. D. Touati-Ahmed, C. Storey. Globally convergent hybrid conjugate gradient methods, J. Optim. Theory Appl. 64, (1990), pp. 379-397.

12. J.C. Gilbert, J. Nocedal. Global convergence properties of conjugate gradient methods for optimization, SIAM J. Optim.2, (1992), pp. $21-42$.

13. L. Zhang, W.J. Zhou, D.H. Li. Global convergence of a modified Fletcher-Revves conjugate gradient method with Armijotype line search, Numer. Math. 104, (2006), pp. 561-572.

14. M.Rivaie, M.Mamat, Leong Wah, Ismail.M. A new class of nonlinear conjugate gradient coefficient with global convergence properties, Appl.Math.comput.218, (2012), pp. 11323-11332.

15. M. Rivaie, A. Abashar, M. Mamat, Ismail. M. The Convergence Properties of a New Type of Conjugate Gradient Methods, Appl. Math. Sciences. Vol. 8, no.1, (2014), pp. $33-44$.

16. A. Abashar, M. Mamat, M. Rivaie, Ismail. M. Global Convergence Properties of a New Class of Conjugate Gradient Method for Unconstrained Optimization, Appl. Math. Sciences. Vol. 8, (2014), pp. 67, 3307 - 3319.

17. N. Andrei. An unconstrained optimization test functions collection, Adv. Modell. Optim. 10, (1977), pp. 147-161.

18. K.E. Hilstrom. A simulation test approach to the evaluation of nonlinear optimization algorithms, ACM. Trans. Math. Softw. 3, (1977), pp. 305-315.

19. E. Dolan, J.J.More. Benchmarking optimization software with performance profile, Math. Prog. 91, (2002), pp. $201-213$. 